uninjured athletes, matched for gender and type of sport, was included.

Assessment of Risk Factors All participants performed a stepping-down task under 4 conditions: 1 . no additional challenges (NORM), 2. whilst performing a cognitive dual-task (DUAL), 3. whilst undergoing unpredictable surface perturbations (PERT), 4. whilst performing a cognitive dual-task + undergoing unpredictable surface perturbations.

Main Outcome Measurements Muscle activations of the vastus medialis (VM), vastus lateralis, hamstrings medialis (HM), hamstrings lateralis were recorded with surface EMG. Integrals were calculated over the landing period (50-250 ms after initial contact) and normalized to maximal voluntary contractions.

Results The ACLR athletes showed an almost unadjusted strategy of increased HM (6.7\% (ACL); 3.9\% (control), $\mathrm{F}=5.07, \mathrm{p}=0.031)$ and decreased $\mathrm{VM}$ activation across all tasks (6.8\% (ACL); $12.9 \%$ (control), $\mathrm{F}=8.52, \mathrm{p}=0.006$ ), whilst the control group had a clear increase in HM activation (3.2\% (NORM); 5.6\% (PERT), t=4.06, $\mathrm{p}=0.001)$ and $\mathrm{VM}$ activation $(9.0 \% \quad(\mathrm{NORM}) ; 16.9 \% \quad(\mathrm{PERT}), \mathrm{t}=3.81$, $\mathrm{p}=0.001$ ) when unpredictable perturbations occurred. However, within the ACLR athletes HM activation decreased when a dual task was added (7.3\% (NORM); 4.7\% (DUAL), $\mathrm{t}=3.4, \mathrm{p}=0.003)$.

Conclusions It seems that the neuromuscular strategy of the ACLR athlete is an overprotective strategy to improve knee stability. They use this strategy in every situation, potentially to compensate for the altered proprioceptive input. This overprotective strategy is jeopardized under cognitively challenging circumstances, confirming that underlying neurocognitive limitations contribute to altered neuromuscular control in ACLR athletes.

\section{GENDER DIFFERENCES IN LANDING MECHANICS AFTER ANTERIOR CRUCIATE LIGAMENT RECONSTRUCTION}

${ }^{1}$ Ahmad Alanazi, ${ }^{2}$ Faisal Al-Enezi, ${ }^{3}$ Mishal Aldaihan, ${ }^{4}$ Hamad Al Amer, ${ }^{5}$ Alexis Ortiz. ${ }^{1}$ Department of Physical Therapy. Majmaah University, Majmaah, Saudi Arabia; ${ }^{2}$ Therapeutic Deputyship, Ministry of Health, Riyadh, Saudi Arabia; ${ }^{3}$ Department of Rehabilitation Health Sciences, King Saud University, Riyadh, Saudi Arabia; ${ }^{4}$ Department of Physical Therapy, University of Tabuk, Tabuk, Saudi Arabia; ${ }^{5}$ UT Health San Antonio, San Antonio, USA

\subsection{6/bjsports-2021-IOC.184}

Background Studies have reported that females and males exhibit different landing techniques. However, few studies have examined the effect of gender on landing technique in athletes following ACLR.

Objective To compare landing technique between females and males following ACLR during landing from heading-jump (LHJ) and landing from long-jump (LLJ).

Design Cross-sectional study.

Setting Biomechanical laboratory.

Patients (or Participants) Eight female and 8 male recreational athletes.

Interventions (or Assessment of Risk Factors) LHJ included jumping forward to head a soccer ball and landing on the force plates, whereas LLJ included jumping forward and landing on the force plates.

Main Outcome Measurements A $2 \times 2$ ANOVA (gender $\times$ landing) was performed to evaluate kinematics, kinetics, and electromyography data.
Results A significant interaction was found only for knee flexion angles $(F 1,14=12.67, p=0.003)$. Pairwise comparisons showed that males landed with decreased knee flexion compared with females during LLJ $(\mathrm{p}=0.01)$. LHJ showed decreased knee flexion compared with LLJ in females $(p<$ $0.001)$ and males $(p=0.001)$. Significant main effects of landing were found. LHJ showed decreased hip flexion angles $(\mathrm{F} 1,14=71.07, \mathrm{p}<0.001)$, decreased knee flexion angles $(\mathrm{F} 1,14=95.17, \mathrm{p}<0.001)$, decreased knee extension moments $(\mathrm{F} 1,14=20.12, \mathrm{p}=0.001)$, and decreased plantarflexion moments $(\mathrm{F} 1,14=34.71, \mathrm{p}<0.001)$. Also, a significant main effect of gender for hip flexion was found showing that males landed with decreased hip flexion angles $(\mathrm{F} 1,14=$ 7.17, $\mathrm{p}=0.01$ ).

Conclusions LHJ showed greater injury predisposing factors compared with LLJ. Females and males following ACLR showed nearly similar landing biomechanics. However, males landed with smaller hip and knee flexion angles (stiff-landing); therefore, preventative training programs may focus on improving the use of hip and knee joints (soft-landing) during landing to decrease the risk of consequent injuries in males following ACLR.

\section{RELATIONSHIP BETWEEN POLE VAULT BIOMECHANICS AND PREVIOUS INJURIES}

1,2,3,4,5 Pascal Edouard, ${ }^{6}$ Hervé Sanchez, ${ }^{3}$ Cyprien Bourrilhon, ${ }^{6}$ Sébastien Homo 7,8 Julien Frère, ${ }^{9,10}$ Johan Cassirame. ${ }^{1}$ Inter-university Laboratory of Human Movement Science (LIBM EA 7424), University of Lyon, University Jean Monnet, Saint-Etienne, France; ${ }^{2}$ Department of Clinical and Exercise Physiology, Sports Medicine Unit, University Hospital of Saint-Etienne, Faculty of Medicine, Saint-Etienne, France; ${ }^{3}$ Medical Commission, French Athletics Federation (FFA), Paris, France; ${ }^{4}$ Swiss Olympic Medical center, Centre de médecine du sport, Division de médecine physique et réadaptation, Centre Hospitalier Universitaire Vaudois, Lausanne, Switzerland; ${ }^{5}$ European Athletics Medical and Anti Doping Commission, European Athletics Association (EAA), Lausanne, Switzerland; ${ }^{6}$ French Athletics Federation (FFA), FIR division, Paris, France; ' Université de Lorraine, Faculty of Medicine, Laboratory " Développement, Adaptation et Handicap » (EA 3450), Vandoeuvre-lès-Nancy, France; ${ }^{8}$ Université de Lorraine, Faculty of Sports Sciences, Villers-lès-Nancy, France; ${ }^{9} E A$ 4660, Culture, Sport, Health and Society Department and Exercise Performance, Health, Innovation platform, University of Bourgogne France Comté, Besançon, France; ${ }^{10}$ EA 7507, Laboratoire Performance, Santé, Métrologie, Société, Reims, France

\subsection{6/bjsports-2021-IOC.185}

Background During pole vault, a high level of energy is transferred from horizontal speed of athlete to the pole. Several ways of performing the pole vault exist, and could be associated with specific injury risk.

Objective To analyse the potential association between the biomechanical patterns of pole vault and the history of injuries.

Design Retrospective study.

Setting National elite indoor championship and youth national indoor championship (U17 and U20) of pole vault (athletics)

Participants $62(70.5 \%$ of the eligible population participating in the championships) healthy national level male and female pole vaulters.

Main Outcome Measurements We prospectively collected the pole vault biomechanical data of the run-up and take-off phases of the vault, and we retrospectively collected the history of injuries during the 12 preceding months through an online questionnaire.

Results A total of $62 \quad(70.5 \%$ of those participating in the championships) pole vaulters accepted to participated in this study, and benefited from pole vault biomechanical and injury 